\title{
Grating and recognition acuities of young amblyopes
}

\author{
M J MOSElEy, A R Fielder, J R THOMPSON, C MINSHUll, ANd D PRICE
}

SUMmary The visual acuities of 36 young amblyopes were determined by $(a)$ conventional recognition tests (near and distance) and $(b)$ an adapted grating acuity card procedure. Considerable agreement between the estimates of acuity obtained with each method was demonstrated, which was generally less than, or equal to, the mean difference between adjacent Snellen lines $\left(4.5 \mathrm{c} \mathrm{deg}^{-1}\right)$. Estimates of grating acuity obtained with vertical gratings did not differ significantly from those obtained with horizontal gratings. There was no difference between the subjects' ability to detect the grating (acuity) and accurately to discriminate target orientation (horizontal or vertical). The results of the experiment are discussed in relation to previous findings of a discrepancy between grating and recognition acuities in amblyopia, and the clinical use of the acuity card procedure.

The observation that infants will preferentially fixate a patterned rather than a 'blank' or homogeneous surface' has spawned a variety of behavioural tests of infant visual acuity. Procedures such as forced-choice preferential looking (FPL) and operant preferential looking (OPL) have been used to study normal visual development ${ }^{2-7}$ and abnormalities of vision in infants and young children. ${ }^{8-11}$ The assessment of visual acuity by preferential looking procedures typically involves the simultaneous presentation of a grating pattern and a homogeneous field of equal spaceaverage luminance. An observer, who is unaware of the position of the stimulus, judges from the infant's looking behaviour whether the grating has been detected. The observer's responses are used to generate a psychometric function from which an estimate of the infant's visual acuity can be obtained. With identical grating targets these procedures can be adapted for use with older children, target detection being signalled by pointing or verbal identification.

Vision tests based on preferential looking have until recently been restricted to laboratory use and have not been employed for routine clinical assessment. The often sophisticated nature of the apparatus required to present the grating stimuli and the need to present a large number of trials to obtain a reliable measure of acuity have limited their clinical application. Recently, however, a new 'acuity card

Correspondence to Mr A R Fielder, Department of Ophthalmology, University of Leicester, Clinical Sciences Building, Leicester Royal Infirmary, PO Box 65, Leicester LE2 7LX. procedure'12 has been developed which may be particularly suitable for routine orthoptic or ophthalmological use. Acuity cards are photographically reproduced square-wave gratings mounted in cardboard frames. The experimenter determines the visual acuity by observing the highest spatial frequency target to which a behavioural response (for example, pointing, eye, and head movements) is elicited. Lengthy psychophysical procedures are not involved. Despite its apparent lack of sophistication the acuity card procedure yields acuity age norms equivalent to those obtained with other more complex forced choice methods and has small interobserver test-retest differences ${ }^{13}$ (Dobson V, Schwartz TL, personal communication).

The assessment of vision with grating targets may be a particularly suitable means of monitoring visual function in young amblyopes undergoing therapy. The use of standard recognition tasks is often inappropriate with an uncooperative, shy, or poorly motivated child or for one who is unable to respond to conventional optotypes. However, there is some evidence to suggest that grating acuities may considerably overestimate recognition acuities in amblyopia; that is, normal grating acuities (6/6 Snellen equivalents) can be recorded in subjects whose recognition acuities are markedly impaired. ${ }^{14}$ One explanation for this finding is the spatial distortion of vision reported in amblyopia' ${ }^{15}$ that would be expected to differentially impair recognition acuity. If the disparity between recognition and grating acuities is consistently large, it may be inappropriate to assess amblyopic vision with grating targets. 
Previous use of the acuity card procedure has been restricted to neonates and infants (typically less than 3 years old). In this study we evaluate the use of an adapted acuity card procedure in an older group of amblyopic children and compare their grating acuities with those obtained by conventional recognition tests.

\section{Materials and method}

Thirty-six children ranging in age from 4.4 to 9.3 years (mean 6.3 years) were recruited for the experiment and were all attending the Orthoptic Department at the Leicester Royal Infirmary. The dragnostic categories of amblyopia in which they were placed were strabismic (17), anisometropic (6), and anisometropic and strabismic (13). The experiment was performed after a review of their orthoptic treatment. Near and distance recognition acuities were measured before and after the determination of grating acuity by a method appropriate to their age and developmental status (Table 1). Grating acuities were measured by an adapted acuity card procedure in which grating targets paired with a 'blank' field of equal space-average luminance were presented within a rectangular aperture in a cardboard surround (Fig. 1). Trials were administered according to a descending method of limits which involved the repetition of each grating three times with position (left, right) randomised. Ten spatial frequencies were tested ranging from $3 \mathrm{c} \mathrm{deg}^{-1}(6 / 60)$ to $30 \mathrm{c} \mathrm{deg}^{-1}$ $(6 / 6)$. Full details of the grating targets are given in Table 1. Testing was terminated at the highest spatial frequency at which three 'don't know' responses were elicited. Subjects were encouraged not to guess.

A total of three grating conditions were presented in a balanced and randomised experimental design. The first two conditions consisted of a simple detection task of gratings which were either vertically (condition 1) or horizontally (condition 2) orientated. In the third condition both horizontal and vertical gratings were presented, and in addition to detecting the presence of the grating subjects were
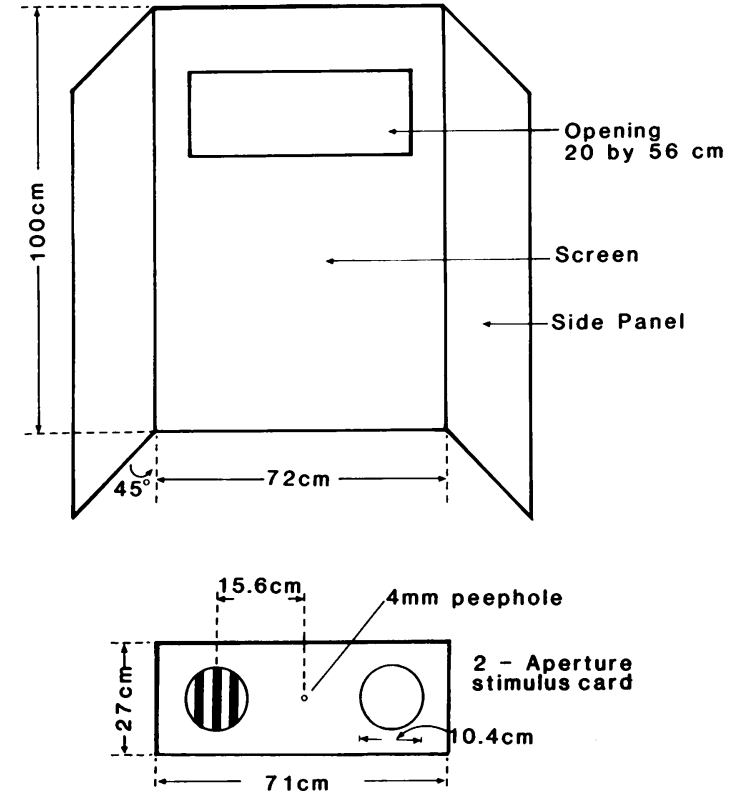

Fig. 1 The acuity card apparatus: surround and stimulus cards. (After McDonald et al. ${ }^{2}$ )

required to indicate its orientation. A variety of behavioural responses were elicited which most commonly included pointing combined with a verbal response. The orientation discrimination task (condition 3) was included because, during clinical use of the acuity card procedure by one of us (ARF), it had been observed that in some cases a considerable disparity existed between a detection threshold (acuity) and the ability to distinguish accurately the orientation of the grating. All acuity measurements were obtained in the amblyopic eye only, with spectacle correction (if prescribed) worn.

\section{Results}

Grating acuities (pooled across horizontal and vertical orientation) were estimated by a logistic

Table 1 Details of grating and recognition acuity tasks

\begin{tabular}{|c|c|c|}
\hline \multicolumn{2}{|l|}{ Recognition acuity } & \multirow[t]{2}{*}{ Grating acuity } \\
\hline Near: $(0 \cdot 33 \mathrm{~m})$ & Distance $(6 m)$ & \\
\hline RAF rule (24) & Snellen: linear optotype (25) & $\begin{array}{l}\text { Grating targets subtended, } 3 \cdot 0,4 \cdot 0,6 \cdot 0,7 \cdot 5,10 \cdot 0,12 \cdot 0,15 \cdot 0,20 \cdot 0 \text {, } \\
24 \cdot 0 \text {, and } 30 \mathrm{c} \mathrm{deg}^{-1} \text { at } 0 \cdot 36 \mathrm{~m}\end{array}$ \\
\hline 'Matching' RAF rule (8) & 'Matching' Snellen: linear optotype (18) & \\
\hline Reduced linear Snellen (4) & Sheridan Gardiner: single optotype (3) & $\begin{array}{l}\text { Acuity cards were back-illuminated with a single tungsten filament light } \\
\text { source. Space-average luminance of targets and 'blank' ficld was } \\
\text { approximately } 1 \cdot 3 \log \mathrm{cd} \mathrm{m}^{-2}\end{array}$ \\
\hline
\end{tabular}




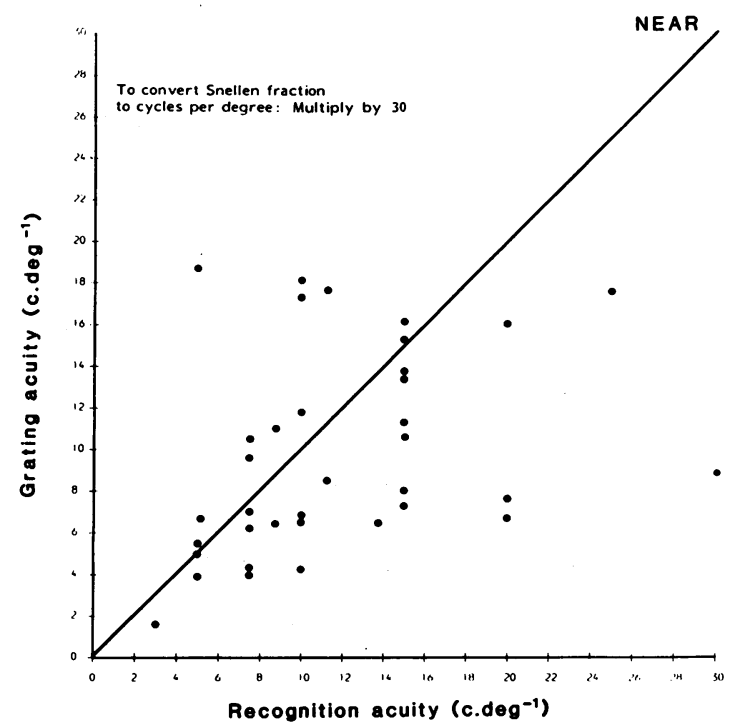

Fig. 2 Relationship between near recognition acuity and grating acuity.

model as described in the Appendix. Near and distance recognition acuity were defined as the arithmetic mean of the scores obtained before and after the determination of grating acuity.

The relationship between grating and recognition acuities (near and distance) are shown in Figs. 2 and 3 . The 'line of equality' corresponds to the points at which grating and recognition acuities are in perfect

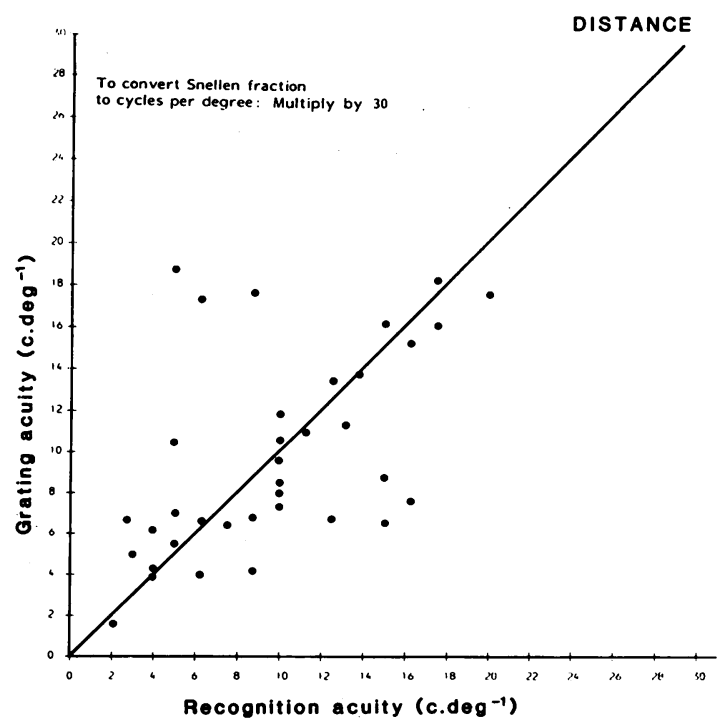

Fig. 3 Relationship between distance recognition acuity and grating acuity.

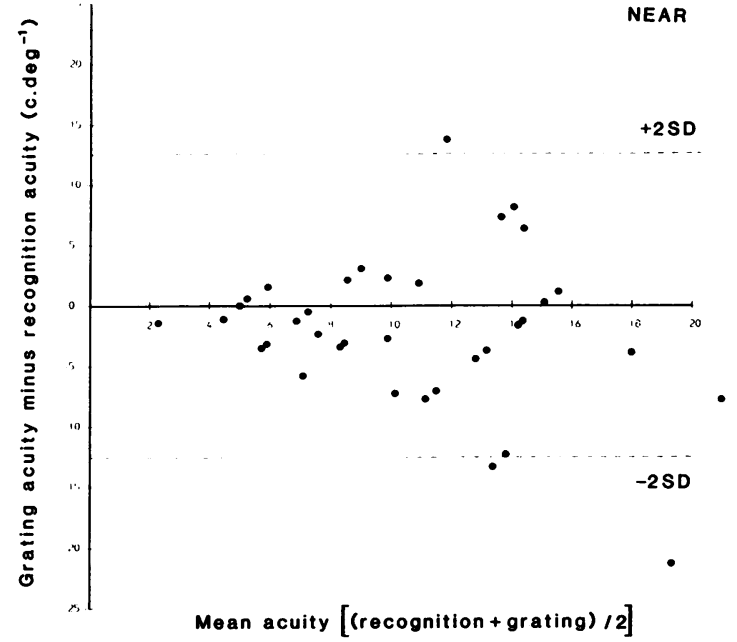

Fig. 4 Difference between grating acuity and near recognition acuity against mean acuity.

agreement. Grating acuity was shown to be significantly correlated with recognition acuity (near: $r=0.33, p<0.05$; distance: $r=0 \cdot 55, p<0 \cdot 001)$.

Although both near and distance recognition acuities are significantly correlated with grating acuity, this finding does not indicate the extent of the agreement between the two measures. The difference between agreement and correlation can be appreciated by inspection of the scattergrams shown in Figs. 2 and 3. Measures of recognition and grating acuity scores are in exact agreement only when they lie on the 'line of equality'. However, a high positive correlation would be obtained if the data points formed any straight line with a positive slope. For a further discussion on the inappropriate use of the correlation coefficient when estimating the agreement between two alternative measures the reader is referred to the recent paper by Bland and Altman. ${ }^{16}$ The extent of the agreement between grating and recognition acuities is shown in Figs. 4 and 5. The data points show for each child the difference between the grating and recognition acuities plotted against the mean acuity (grating+recognition/2). Perfect agreement is indicated by points falling on the 'zero difference line'-agreement decreasing as a function of the distance between this line and each data point.

The final analysis compared the grating detection thresholds (acuity) with the thresholds obtained for correctly discriminating the orientation of the grating targets (see 'Materials and methods'). A $\chi^{2}$ test of the change in the log likelihood (see Appendix) failed to reveal any difference between the subjects' ability to detect or discriminate the orientation of the gratings. 


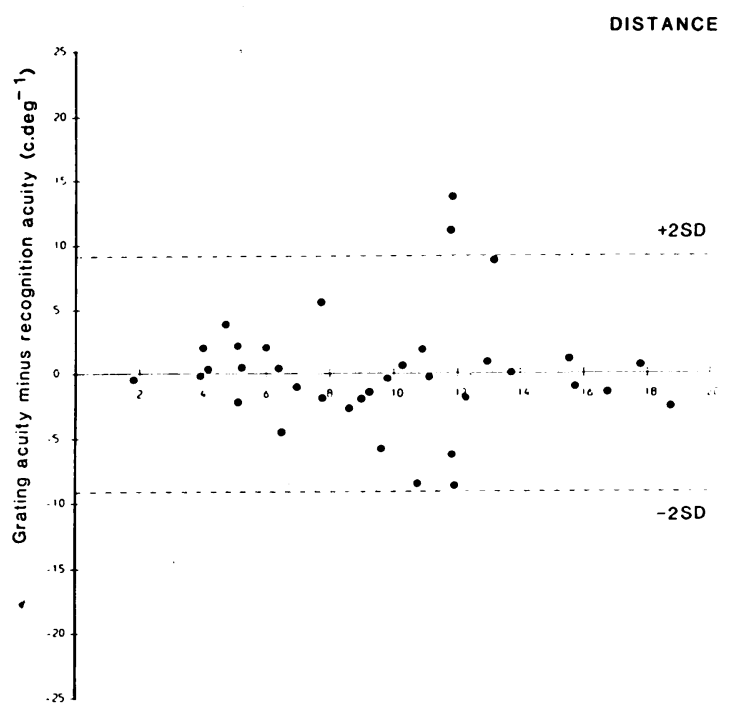

Mean acuity [(recognition+grating)/2]

Fig. 5 Difference between grating acuity and distance recognition acuity against mean acuity.

Similarly, there were no differences in the grating acuities obtained with horizontal or vertical gratings.

\section{Discussion}

If the acuity card procedure is to prove a viable method of routinely assessing visual acuity in amblyopes it must fulfil two criteria. Firstly, grating acuities must relate in some predictable manner to those obtained with conventional methods. Secondly, the ease and length of time taken to administer the test must be in accord with the constraints imposed by clinical use.

The extent of the agreement between grating and recognition acuities is shown in Figs. 4 and 5. Adopting a bandwidth of $4.5 \mathrm{c} \mathrm{deg}^{-1}$ (this corresponds to the mean difference between adjacent lines on a standard Snellen chart in the range $6 / 6$ to $6 / 60$ ) we can see that $24(67 \%)$ of the differences between the grating and recognition acuities against the mean acuities fall on or within this bandwidth for near recognition and $28(78 \%)$ for distance recognition. Apart from cases in which distance acuity was determined from single optotype characters, the proportion of scores that fall within the $4.5 \mathrm{c} \mathrm{deg}^{-1}$ bandwidth rises to $82 \%$-that is, the grating and recognition acuities of 27 out of 33 subjects agreed within one mean Snellen line (single optotypes will frequently overestimate recognition acuity owing to the 'crowding effect' or contour interaction that occurs with line optotypes). ${ }^{17}$ Ix Doubling this bandwidth to $9 \mathrm{c} \mathrm{deg}^{-1}$ that is, twice the mean difference between adjacent Snellen lines, leaves 32(89\%) of near recognition acuities and $34(94 \%)$ of distance recognition acuities within this bandwidth. These findings show that grating acuities can provide clinically useful estimates of visual acuity which are, in most cases, in good agreement with those obtained by conventional methods.

The results failed to reveal any differences between grating detection thresholds (acuity) and the threshold for correctly discriminating grating orientation. It had been supposed from our previous clinical experience that the orientation discrimination task would prove more difficult. Careful attention was paid to matching the luminance of each grating target with that of the corresponding 'blank' by back-illuminating the acuity cards. Failure to do this could have resulted in the child responding to the brightest aperture in the test card, thus producing a spurious estimate of acuity. Our experience suggests that equating the luminance of the grating targets with the 'blank' field is an important prerequisite for the efficient administration of the acuity card procedure. Incorporating an orientation discrimination task into the acuity card procedure may be one safeguard to prevent subjects from responding to differences in luminance when presented with a subthreshold grating target.

Our results do not confirm previous findings ${ }^{1014}$ that grating acuities will significantly overestimate recognition acuity-none of our sample of amblyopic children was found to possess a normal grating acuity

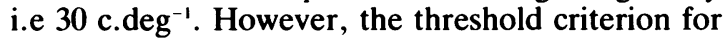
grating acuity (probability of correct detection $=95 \%$, see Appendix) was deliberately set to a high level. This ensured that grating acuity was appropriately equated with the high level of certainty with which the recognition acuities were obtained in this study and indeed in standard clinical practice. Using a slightly less rigorous $(80 \%)$ criterion, Jenkins and co-workers ${ }^{19}$ have also demonstrated the predictive value of grating acuity in the assessment of amblyopic vision. In clinical practice however, the subjective method of analysis of the acuity card procedure may increase the likelihood of grating acuities exceeding those obtained with a recognition task.

In this study grating acuities were determined by two orthoptists with considerable experience in measuring visual acuity in young children. In their opinion the adapted acuity card procedure was easy to administer, and frequently a more rapid response was elicited from the child presented with the acuity cards than when presented with a recognition target. The test procedure for determining grating acuities in the present experiment differed from that originally described. Here, acuities were obtained with a 
descending method of limits in order that the possible differences in thresholds for gratings of different orientations could be accurately determined. (In the original version of the test the examiner is free to choose which gratings to present and the estimate of acuity is based on their subjective opinion of the infant's behaviour.) This adaptation inevitably increased the time taken to administer the test, though it was apparent that, had the original acuity card procedure been adhered to, an estimate of acuity could have been obtained in 2 to 3 minutes.

\section{APPENDIX}

\section{The modified logistic model}

The usual logistic model for analysing binary data assumes that there exists a threshold above which the subject's response is correct and below which it is incorrect. When performing the experiment the examiners noticed that despite counter instruction many of the children appeared to guess the position of the grating, an observation supported by the number of incorrect replies given. To allow for this in the analysis the logistic model was modified to allow for three responses, namely 'correct', 'incorrect', and 'don't know'.

The modified logistic model assumes that there exist thresholds $q_{0}$ and $q_{1}$. Below $q_{0}$ the response is 'correct', between the two the subject guesses and thus has an equal chance of being 'correct' or 'incorrect', and above $q_{1}$ the response is 'don't know'.

If $\mathrm{F}$ represents the distribution function of the logistic distribution the probabilities of the three possible responses may be expressed as,

$$
\begin{aligned}
& \text { Prob. (correct) })=1 / 2\left[F\left(\mathrm{q}_{1}-\mathrm{by}\right)+\mathrm{F}\left(\mathrm{q}_{0}-\mathrm{by}\right)\right] \text {, } \\
& \text { Prob. (incorrect })=1 / 2\left[\mathrm{~F}\left(\mathrm{q}_{1}-\mathrm{by}\right)-\mathrm{F}\left(\mathrm{q}_{0}-\mathrm{by}\right)\right] \text {, } \\
& \text { Prob. (don't know })=1-\mathrm{F}\left(\mathrm{q}_{1}-\mathrm{by}\right),
\end{aligned}
$$

where $y$ is the size of the grating under test and $b$ is a scale parameter.

The parameters $q_{0}, q_{1}$, and $b$ were estimated by the method of maximum likelihood, and the grating acuity $y_{0}$ of the subject was defined as that grating that they would have correctly located on $95 \%$ of tests. That is to say,

$$
1 / 2\left[F\left(q_{1}-b y_{0}\right)+F\left(q_{0}-b y_{0}\right]=0 \cdot 95\right. \text {. }
$$

The authors acknowledge the co-operation of Ms Elizabeth Bacon in providing the facilities in the Orthoptic Department at the Leicester Royal Infirmary, and the helpful comments provided by Dr Luisa Mayer on a previous draft of this paper.

\section{References}

1 Fantz RL. Pattern vision in young infants. Psychological Record 1958; 8: 43-7.
2 Teller DY, Morse R. Borton R, Regal D. Visual acuity for vertical and diagonal gratings in human infants. Vision Res 1974; 14: 1433-9.

3 Dobson V, Teller DY, Belgum J. Visual acuity in human infants assessed with stationary stripes and phase-alternated checkerboards. Vision Res 1978; 18: 1233-8.

4 Atkinson J, Braddick O, Pimm-Smith E. 'Preferential looking' for monocular and binocular acuity testing of infants. $\mathrm{Br} J$ Ophthalmol 1982; 66: 264-8.

5 Mayer DL, Dobson V. Assessment of vision in young children: a new operant approach yields estimates of acuity. Invest Ophthalmol Vis Sci 1980; 19: 566-70.

6 Mayer DL, Dobson V. Visual development in infants and young children as assessed by operant preferential looking. Vision Res 1982; 22: 1141-51.

7 Sokol S, Moskowitz A. Comparison of pattern VEPs and preferential-looking behaviour in 3-month-old infants. Invest Ophthalmol Vis Sci 1985; 26: 359-65.

8 Jacobson SG, Mohindra I, Held R. Visual acuity of infants with ocular diseases. Am J Ophthalmol 1982; 93: 198-209.

9 Mayer DL, Fulton AB, Hansen RM. Preferential looking acuity obtained with a staircase procedure in pediatric patients. Invest Ophthalmol Vis Sci 1982; 23: 538-43.

10 Mayer DL, Fulton AB, Rodier D. Grating and recognition acuities of pediatric patients. Ophthalmology 1984; 91: 947-53.

11 Mayer DL, Fulton AB. Preferential looking grating acuities of infants at risk of amblyopia. Trans Ophthalmol Soc UK 1985; 104: 903-11.

12 McDonald M, Dobson V, Sebris SL, Baitch L, Varner D, Teller DY. The acuity card procedure: a rapid test of infant visual acuity. Invest Ophthalmol Vis Sci 1985; 26: 1158-62.

13 Teller DY, McDonald M, Preston K, Sebris SL, Dobson V. Visual acuity in infants and children: the acuity card procedure. Dev Med Child Neurol 1986; 28: 779-89.

14 Howell ER, Mitchell DE, Keith CG. Contrast thresholds for sine gratings of children with amblyopia. Invest Ophthalmol Vis Sci 1983; 24 : 782-7.

15 Hess RF, Campbell FW, Greenhalgh T. On the nature of the abnormality in human amblyopia; neural aberrations and neural sensitivity loss. Pflügers Arch 1978; 377: 201-7.

16 Bland JM, Altman DG. Statistical methods for assessing agreement between two methods of clinical measurement. Lancet $1986 ;$ i: $307-10$.

17 Stuart JA, Burian HM. A study of separation difficulty; its relationship to visual acuity in normal and amblyopic eyes. $A m J$ Ophthalmol 1962; 53: 471-7.

18 Keith CG, Howell ER, Mitchell DE, Smith S. Clinical trial of the use of rotating grating patterns in the treatment of amblyopia. Br J Ophthalmol 1980; 64: 597-606.

19 Jenkins PL, Simon JW, Kandel GL, Forster T. A simple grating acuity test for impaired children. Am J Ophthalmol 1985; 99: 652-8.

Accepted for publication 29 May 1986. 